\section{Scientists uncover potential new method of tooth repair}

An international team of researchers has found a new mechanism that could offer a potential new solution to tooth repair. ${ }^{1}$ They discovered a new population of mesenchymal stromal cells in a continuously growing mouse incisor model. They have shown that these cells contribute to the formation of dentine.

The study was led by Dr Bing Hu of the Peninsula Dental School of the University of Plymouth, UK.

Importantly, the work showed that when the mesenchymal stromal cells are activated they send signals back to the mother cells of the tissue to control the number of cells produced, through a molecular gene called Dlk1. This study is the first to show that Dlk1 is vital for this process to work. In the same study, the researchers also demonstrated that Dlk1 can enhance stem cell activation and tissue regeneration in a wound healing model. This mechanism could provide an innovative solution for tooth repair, addressing problems such as tooth decay, crumbling

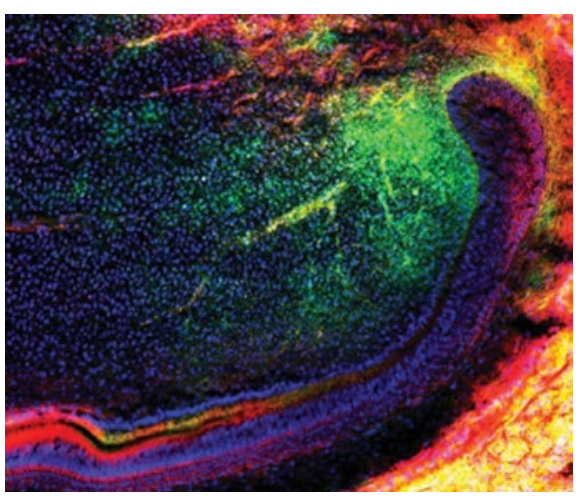

A group of mesenchymal (green) stem cells migrating in a tooth to further regenerate tissues. Source: Media and Communications, University of Plymouth

and trauma treatment. Further studies are needed to validate the results for clinical applications to determine the appropriate duration and dose of treatment.

Dr Denis Corbeil from the Biotechnology Center of the TU Dresden (BIOTEC), one of the co-authors of the study, said: 'The discovery of this new population of stromal cells was very exciting and has enormous potential in regenerative medicine.

\title{
Orthodontic treatment for adults continues popularity
}

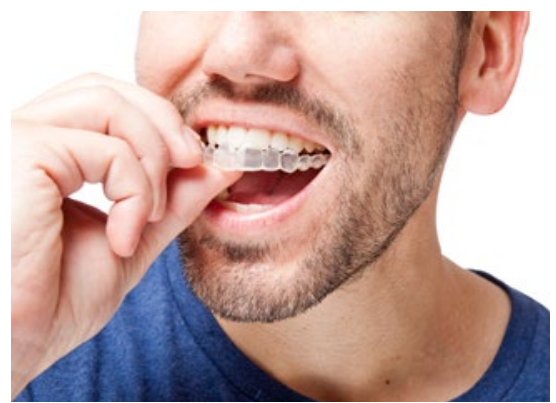

New figures released by the British Orthodontic Society (BOS) has revealed that the number of adults seeking orthodontic treatment in the UK remains high. The survey, conducted in July 2019 among BOS members, was designed to gather new data about orthodontics and patient choices in the UK.

Asked if they were seeing an increase in private adult treatment, $75 \%$ said yes. The majority of adult patients (80\%) are in the
26 to 55 age bracket. The survey revealed that adult patients are most likely to be female (80\%). However, the number of men seeking treatment appears to be on the rise. Twenty percent of the respondents to the survey estimate that half of their adult patients are male.

When asked what kind of braces they provide to their patients, orthodontists revealed a cross section of approaches:

- Over $75 \%$ supply fixed braces with clear aesthetic brackets

- Over $30 \%$ supply lingual braces

- $70 \%$ supply clear aligners.

The most popular system, provided by more than $98 \%$ of orthodontists, is fixed braces on the front of the teeth. This figure reflects the high number of young people treated as NHS patients for whom fixed braces is the most appropriate option.
Faculty of Dental Surgery to offer new endodontic webinars

The Royal College of Surgeons of Edinburgh (RCSEd) is launching its first series of dental webinars that will focus on key principles of endodontics: access, instrumentation and obturation. These free resources offer solutions to gaps in dental education and aid dental trainees. They are open to all during the live stream (registration required) then archived so RCSEd membership can access them long-term.

The RCSEd offers support and resources to dentists at all stages of their career through its Faculty of Dental Surgery and Faculty of Dental Trainers, from its Dental Skills Competition for trainees (launching Aug/Sept 2019) to its Standards for Dental Trainers, to ensure consistent treatment and support patient safety. RCSEd introduced its surgical webinars in 2013 and has delivered 154 sessions to 5,500 participants.

Each webinar will be presented by a specialist endodontist with a focus on the technical aspects of the various treatment procedures and the importance of underpinning this with sound biological knowledge. While they mainly focus on dental trainees, the webinars offer technique tips to improve efficiency or can be used to refresh core knowledge in endodontics.

Dental Education Convenor Dr Brian Nattress said: 'The College is delighted to launch this first series of webinars focusing on endodontic treatment. Endodontics is a constantly changing field of dentistry with the introduction of a number of innovations in both techniques and instruments used for diagnosis and treatment. Our speakers have considerable expertise in treating and teaching all aspects of the speciality and will provide a background of the scientific basis of the subject along with a number of practical tips'.

Registration is open at rcsed.ac.uk/ webinars. 